\title{
Third order non-linear optical properties of L-arginine hydrochloride monohydrate single crystals by Z-scan technique
}

\author{
V. Natarajan ${ }^{1}$, T. Sivanesan ${ }^{2}$ and S. Pandi ${ }^{3}$ \\ ${ }^{1}$ Dept. of Physics, Rajalakshmi Institute of Technology, Kunthampakkam-602124, TN, India \\ ${ }^{2}$ Dept. of Physics, Aksheyaa College of Engineering, Puluthivakkam, Kanchipuram 603 314, TN, India \\ ${ }^{3}$ Dept. of Physics, Presidency College, Chennai- 600 005, India.
}

hinatarajan@gmail.com

\begin{abstract}
L-arginine hydrochloride monohydrate (L-arginine hydrochloride monohydrate) was synthesized by the reaction between hydrochloric acid and the strongly basic amino acid, L-arginine taken in the ratio of 1:1. The crystal structure of the sample was studied by single X-ray diffraction. The UV transmittance spectra of the grown crystals indicate a good transparency between 200 and $1000 \mathrm{~nm}$. The nonlinear refractive index $n_{2}$ and susceptibility $x^{(3)}$ have been measured through the Z-scan technique. The results indicate that the compound exhibits reverse saturation absorption and selfdefocusing performance. Non-linear absorption co-efficient is determined as $9.2194 \times 10^{-4} \mathrm{~cm} / \mathrm{w}$. The non-linear refractive index of the L-arginine hydrochloride single crystals were found to be $1.7129 \times 10^{-7} \mathrm{~cm}^{2} / \mathrm{w}$. The real and imaginary pars of non-linear susceptibility $x^{(3)}$ have been measured at $632.8 \mathrm{~nm}$ and found to be $1.1573 \times 10^{-05}$ esu and $3.1382 \times 10^{-65}$ esu, respectively.
\end{abstract}

Keywords: Growth from solution, single XRD, UV spectrum, Z-scan

\section{Introduction}

L-arginine hydrochloride monohydrate is one of the new semi organic NLO materials by Monaco et al. (1987). Use of these crystals for NLO devices demands large size single crystals of extremely high quality. Here we present the third order non-linear optical properties of Larginine hydrochloride monohydrate by Z-scan technique. The Z-scan technique (Zhao et al., 1993; Yin et al. 2000; Natarajan et al., 2010; Sivanesan et al., 2010) is a popular method for the measurement of optical nonlinearities of materials. It has the advantage of high sensitivity and simplicity. One can simultaneously measure the magnitude and sign of the non-linear refraction and non-linear absorption, which are associated with the real part $X_{R}{ }^{(3)}$ and imaginary part $X_{1}{ }^{(3)}$ of the third order non-linear susceptibilities. The Z-scan technique has been used to measure the non-linear optical properties of semiconductors (Krauss \& Wise, 1994), dielectrics (Gomes \& De Araujo, 1992; RangelRojo, et al., 1994) organic or carbon-based molecules (Wei et al., 1992; Zhang et al., 1993) and liquid crystals (Paparo et al., 1994; Li et al., 1994). In this work we present the growth of L-arginine hydrochloride monohydrate single crystals from aqueous solution. Single X-ray diffraction, optical absorption spectrum Z-scan measurements were carried out. Z-scan results reveal that Larginine hydrochloride monohydrate single crystal is a potential candidate for the optical switching (Lee et al., 1993) and optical limiting (Fryad et al., 1993).

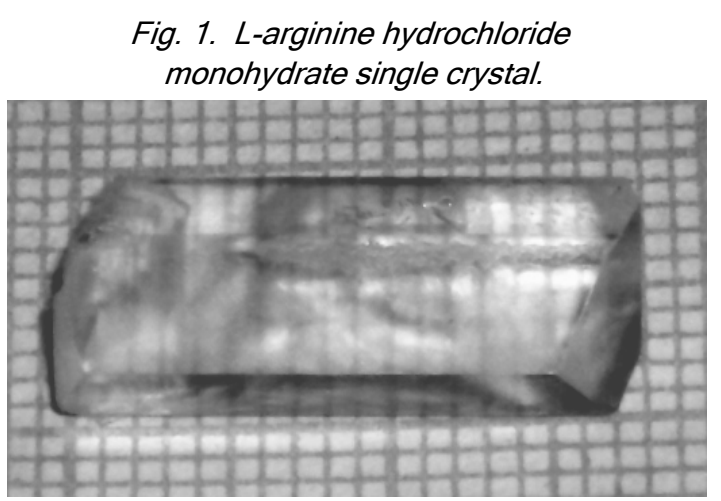

"Synthesis of L-arginine single crystal" http://www.indjst.org

\section{Crystal growth}

Synthesis of L-arginine molecule $\mathrm{NH}_{2} \mathrm{CNHNH}\left(\mathrm{CH}_{2}\right)_{3}$ $\mathrm{CH}\left(\mathrm{NH}_{2}\right) \mathrm{COOH}$ has two groups namely guanidyl and amino groups, which can be protonated. L-arginine hydrochloride monohydrate single crystal was synthesized by the reaction between hydrochloric acid and the strongly basic amino acid, L-arginine taken in the ratio of 1:1 (higher ratios like two or more yields highly viscous solution). The required amount of hydrochloric acid was added to the double distilled water. Then the calculated amount of L-arginine was added and dissolved in dilute hydrochloric acid. The following reaction takes place in this process

$\mathrm{NH}_{2} \mathrm{CNHNH}\left(\mathrm{CH}_{2}\right)_{3} \mathrm{CH}\left(\mathrm{NH}_{2}\right) \mathrm{COOH}+\mathrm{HCl}+\mathrm{H}_{2} \mathrm{O} \rightarrow$ $\left(\mathrm{H}_{2} \mathrm{~N}\right)_{2}{ }_{2}^{+} \mathrm{CNH}\left(\mathrm{CH}_{2}\right)_{3} \mathrm{CH}\left(\mathrm{NH}_{3}\right)^{+} \mathrm{COO}^{-} \mathrm{Cl}^{-} \mathrm{H}_{2} \mathrm{O}$.

The synthesized salt was purified by repeated recrystallization process until optically clear crystals of size $1.9 \times 0.9 \times 0.4 \mathrm{~mm}^{3}$ was obtained as shown in Fig. 1 . Then it was grounded to around $2 \mathrm{~mm}$ thickness.

Results and discussion

Single crystal XRD

Single crystal $X$-ray diffraction analysis was carried out to determine the lattice parameters. This study reveals that the grown crystal belongs to monoclinic system with the space group $P_{21}$. The calculated lattice parameter values are listed in Table 1. The single crystal XRD results are in good agreement with the reported values and thus confirm the structure of the grown crystal.

\section{Optical measurements}

\section{Optical absorption spectrum: The}

Natarajan et al. Indian J.Sci.Technol. 
optical absorption spectrum analysis of the grown crystal was carried out between $200 \mathrm{~nm}$ to $900 \mathrm{~nm}$ using Varian Cary 5E spectrophotometer. The recorded spectrum is as shown in the Fig. 2. The crystal is transparent in the entire visible region and shows maximum UV transmission. The L-arginine hydrochloride monohydrate possesses a good transparency of $74 \%$ with the lower cut-off wavelength at $220 \mathrm{~nm}$. The material can find application as windows in spectral instruments in this region.

Refractive index measurement: The refractive index of the L-arginine hydrochloride monohydrate crystal was determined by Brewster's angle method using He-Ne laser of wavelength $632.8 \mathrm{~nm}$. A polished flattened single crystal of L-arginine hydrochloride monohydrate was mounted on a rotating mount at an angle varied from 0 to $90^{\circ}$. The angular reading on the rotary stage was observed, when the crystal is perfectly perpendicular to the incident beam. The crystal was rotated until the laser oscillates and the angle has been set for maximum power output. Brewster's angle $(\theta p)$ for L-arginine hydrochloride monohydrate is measured to be $58.5^{\circ}$. The refractive index has been calculated using the equation $n=\tan \theta p$; where $\theta p$ is the polarizing angle and it is found to be 1.632.

\section{Non-linear optical measurements}

SHG measurements: The second harmonic generation efficiency of the grown crystals was carried out using the Kurtz powder technique (Kurtz \& Perry et al., 1968). The sample is irradiated at $1064 \mathrm{~nm} \mathrm{Nd:} \mathrm{YAG} \mathrm{pulsed} \mathrm{laser.}$ The crystal of L- arginine hydrochloride monohydrate single crystals were powdered with a particle size of 100 $150 \mu \mathrm{m}$ and then placed in a micro capillary and exposed to laser radiation. The output from the sample was monochromatic to collect the intensity of $532 \mathrm{~nm}$ component, and to eliminate the fundamental wavelength. Second harmonic radiation generated by the randomly oriented micro crystals was focused by a lens and detected by a photo multiplier tube. The generation of the second harmonic was confirmed by the emission of green light. Urea crystal was powdered to the same particle size as the experimental sample and it was used as a reference material for this measurement. The SHG conversion efficiency of L-arginine hydrochloride monohydrate single crystal was found to be 0.17 with respect to Urea and 0.38 times that of KDP it is comparable with the reported values (Meera et al., 2004; Kalaiselvi et al., 2008). Thus, $\Pi-\Pi^{*}$ transitions occurs in the carboxylate and guanidyl groups which give rise to the NLO properties in of L- arginine hydrochloride monohydrate single crystal.

Z-scan method: The third order optical non-linearities of L-arginine hydrochloride monohydrate single crystals
Vol. 3 No. 8 (Aug 2010)

ISSN: 0974- 6846
Table 1. Single XRD data of L-arginine hydrochloride monohydrate single crystals Formula wt. 274.66 Space group $\mathrm{P}_{21}$ $+$ were investigated with the Z-scan method as outlined earlier (Sivanesan et al., 2010). A CW HeNe laser of wavelength of $632.8 \mathrm{~nm}$ was used in the experiment. The laser beam was focused to a waist of $45 \mu \mathrm{m}$ with the help of a convex lens of focal length $12 \mathrm{~cm}$ to give the intensity at the focus $6.25 \times 10^{7}$ $\mathrm{W} / \mathrm{m}^{2}$. The sample is moved along the (beam direction) optic axis (Zdirection) through the focus of the lens. The energy transmitted through an aperture is recorded as a function of the sample position. The Rayleigh length of $Z_{0}$ was calculated is greater than the thickness of the sample. Fig. 3 shows the normalized transmission for the open aperture $(\mathrm{OA})$.

The transmission is symmetric with respect to the focus $(Z=0)$ where it has a minimum transmission. This indicates that the sample exhibits resonance saturation absorption (RSA/two photon absorption). Phase shift at the focus is calculated using the relation

$\Delta \Phi_{0}=\beta I_{0} L_{\text {eff }} / Z$

Leff $=[1-\exp (-\alpha d) / \alpha]$ where $\alpha$ is the linear absorption coefficient at $632.8 \mathrm{~nm}$ and "d" is the thickness of the sample. $L l_{\text {eff }}$ is the effective thickness of the sample $I_{0}$ is the intensity of the beam focused at the focus and $\beta$ is non-linear absorption co-efficient. $\beta$ is calculated from the formula:

$\beta=2 \sqrt{ } 2 \Delta T / I_{0} L_{\text {eff }}$

The calculated value of $\beta=9.2194 \times 10^{-4} \mathrm{~cm} / \mathrm{W}$. The imaginary part of the third order non-linearity $x_{l}^{(3)}$ is related to the non-linear absorption co-efficient $\beta$ by the relation

$X_{1}^{(3)}=10^{-2} \epsilon_{0} c^{2} n_{0}{ }^{2} \lambda \beta / 4 \Pi^{2}$

Where $n$ is the linear refractive index $(n=1.453$ at $\lambda=$ $632.8 \mathrm{~nm}) \varepsilon$ is the permittivity of free space and $c$ is the velocity of light. The experimentally determined value of $x$ $1^{(3)}$ at $632 \mathrm{~nm}$ was found to be $3.1382 \times 10^{-05} \mathrm{esu}$. The normalized transmittance for closed aperture (CA) is observed in Fig. 4.

The non-linear refractive index of the said crystal is calculated using the relation

$$
\text { ' } \mathrm{n}_{2 "}=\Delta \Phi_{0} / \mathrm{KI}_{0} \mathrm{~L}_{\text {eff }}
$$

The peak to valley configuration of the curve suggests that the refractive index change is negative, exhibiting a self defocusing effect. This may be an advantage for the application in protection of optical sensors. The value for non-linear refractive index " $\mathrm{n}_{2 \text { " }}$ calculated by $z$-scan method is found to be $1.7129 \times 10^{-7} \mathrm{~cm}^{2} \mathrm{~W}$.

The real part of the third order non-linear susceptibility $X_{R}{ }^{(3)}=2 n_{0}^{2} \varepsilon c Y$

The value of of $X_{R}{ }^{(3)}$ at $632 \mathrm{~nm}$ is found to be $1.1573 \mathrm{x}$ $10^{-05}$ esu. By comparing the values of imaginary and real non-linearities, one can come to the conclusion that the $X$ ${ }^{(3)}>X_{R}{ }^{(3)}$, i.e. $X{ }^{(3)}$ which gives rise to the absorption
Research communication

(CIndian Society for Education and Environment (iSee)
"Synthesis of L-arginine single crystal" http://www.indjst.org
Natarajan et al. Indian J.Sci.Technol. 
change, is dominant, and this can be seen from Fig. (3) where the valley is much larger than the peak. The absolute value of $\left|X^{(3)}\right|$ was calculated from the formula:

$\left|X R^{(3)}\right|=\sqrt{\left(X_{1}{ }^{(3)}\right)^{2}+\left(X_{R}{ }^{(3)}\right)^{2}}$ gives $3.3448 \times 10^{-05}$ esu. The value of the $X_{1}{ }^{(3)}$ and $X_{R}{ }^{(3)}$ of Larginine hydrochloride monohydrate reported here is of the same order of the magnitude of the materials such as Chalogenide glasses (Sheik-Bahe et al., 1990) and $\mathrm{C}_{60}$ (Fryad et al., 1993) etc.

Conclusion

We have reported the optical properties of L-arginine hydrochloride monohydrate single crystal. The Z-scan measurement with $632.8 \mathrm{~nm}$ laser pulses revealed that nonlinear refractive index of the crystal is in the range of $10^{-7}$ $\mathrm{cm}^{2} / \mathrm{W}$. The measured $3^{\text {rd }}$ order non-linear properties confirm its suitability for non-linear optical devices such as optical limiting (Lee et al., 1993) and optical switching (Fryad et al., 1993).

\section{Acknowledgements}

The authors thank Prof. Sastikumar, NIT, Tiruchi for providing the Z-scan facility and Prof. P.K.Palanichamy \& Mr. Ahamed, Anna Univ. for their valuable suggestions.

\section{References}

1. Fryad Z Henari, Shane MacNamara, Stevenson O, Callagham J, Weldon $\mathrm{D}$ and Blau WJ (1993) Low power nonlinear optical response of C60 and C70 fullerene solutions. Adv. Mater. 5, 930-936.

2. Gomes MH and De Araujo GB (1992) Infrared non-linearity of commercial $\mathrm{Cd}(\mathrm{S}, \mathrm{Se})$ glass composites. Opt. Commun. 87, 19-22.

3. Kalaiselvi D, Mohan Kumar R and Jayavel R (2008) Single crystal growth and properties of semi-organic nonlinear optical L-arginine hydrochloride monohydrate crystals. Cryst. Res. Technol. 43(8), 851-856.

4. Kurtz SK and Perry TT (1968) A powder technique for the evaluation of non-linear optical materials. J. Appl. Phys. 39, 3798-3812.

5. Lee W Tutt and Boggess TF(1993) A review of optical limiting mechanisms and devices using organics, fullerenes,

Vol. 3 No. 8 (Aug 2010)

ISSN: 0974- 6846

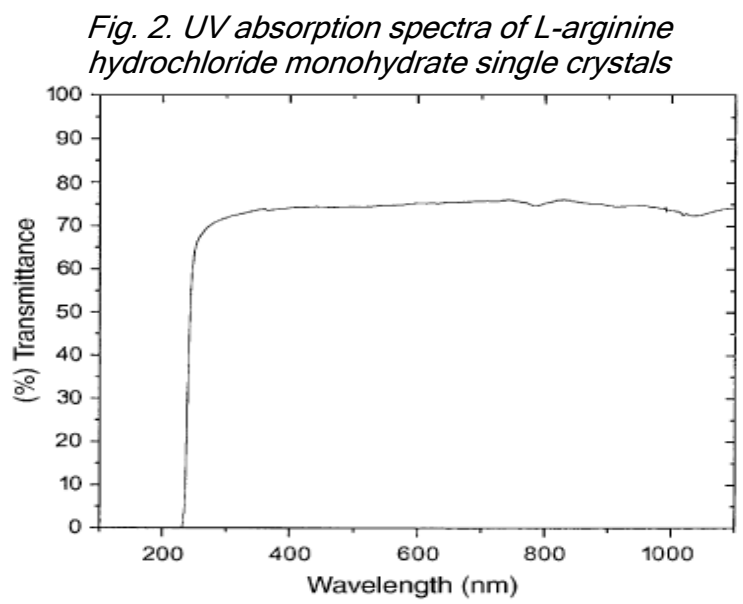

Fig. 3. Open aperture curve of L-arginine

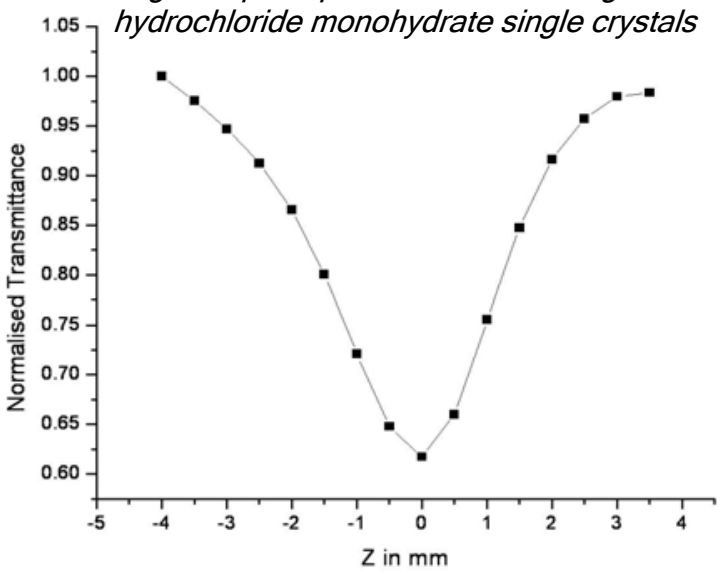

Fig. 4. Closed Aperture curve of L-arginine

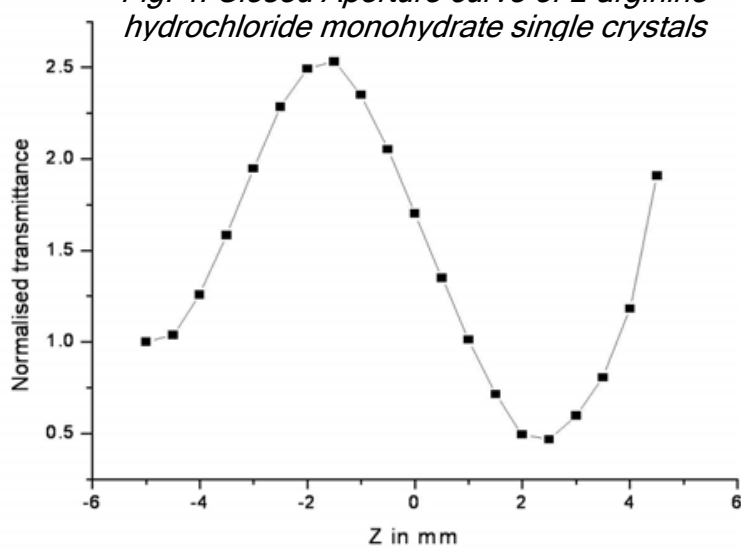

semiconductors

and other materials. Prog. Quant. Elecr. 17, 299-306.

6. Li L, Yuan HJ, Hu GH and PalffyMuthoray $P$ (1994) A new configurational transition in inhomogeneous nematics. Liq. Cryst. 16, 703-712.

7. Meera K, Muralidharan R, Dhanasekaran $\mathrm{R}$, Manyum $\mathrm{P}$ and Ramasamy P (2004) Growth of non-linear optical material: Larginine hydrochloride and its characterization. J. Crystal Growth. 263, 510-516.

8. Monaco SB, Davis LE, Velso SP, Wang FT, Eimerl D and Zalkin A (1987) Synthesis and characterization of chemical analogs of L-arginine phosphate. J. Crystal Growth. 85, 252- 257.

9. Natarajan V, Sivanesan $T$ and Pandi S (2010) Third order nonlinear optical properties of potassium aluminium sulphate single crystals by Z-Scan technique. Indian J.Sci.Technol. 3 (6), 656-658.

10. Paparo D, Maddalena P, Abbade G, Santamato $E$ and Jannossy (1994) Wavelength dependence of optical reorientation in dye-doped nematics. Mol. Cryst. Liq. Cryst. 251, 73-84.

11. Rangel-Rojo R, Kosa T, Hajito E, Ewen PJS, Owen AE, Kar AK and Whereett BS (1994) Near-infrared optical non-linearities in amorphous chalcogenides. Opt. Commun. 109, 145-150.

12. Sivanesan T, Natarajan $V$ and Pandi S (2010) Non-linear optical properties of a-glycine single crystals by Z-Scan technique. Indian J.Sci.Technol. 3 (6), 653655.

13. Tod D Krauss and Wise FW (1994)Femto-second measurement of non-linear absorption and refraction in CdS, ZnSe, and ZnS. Appl. Phys. Lett. 65, 1739-1741.

14. Wei TH, Hagan DJ and Sence MJ, Van Stryland EW, Perry JW and Coulter DR (1992) Direct measurements of non-linear absorption and refraction in solutions of phthalocyanines. Appl. Phys. B. 54, 46-51.

15. Yin M, Li HP, Tang SH and Ji W (2000) Determination of non-linear absorption and refraction by single Z-scan method. Appl. Phys. B. 70, 587-591.

16. Zhang W, Zen H, Du Y, Han Y, Dong F and Xia Y (1993) Large non-linear absorption in C60 thin films. J. Phys. B. 26, L451-L456.

17. Zhao W and Palffy-Muhoray P (1993) Z-scan technique using top-hat beams. Appl. Phys. Lett. 63, 1613-1615. 